\title{
Adipose Stromal Cells from Visceral and Subcutaneous Fat Facilitate Migration of Ovarian Cancer Cells via IL-6/JAK2/STAT3 Pathway
}

\author{
Boyun Kim, $\mathrm{PhD}^{1,2}$ \\ Hee Seung Kim, MD, PhD ${ }^{3}$ \\ Soochi Kim, MS ${ }^{1}$ \\ Guy Haegeman, $\mathrm{PhD}$ \\ Benjamin K. Tsang, $\mathrm{PhD}^{4}$ \\ Danny N. Dhanasekaran, $\mathrm{PhD}^{5}$ \\ Yong Sang Song, MD, $\mathrm{PhD}{ }^{1,3,6}$
}

\section{${ }^{1}$ Cancer Research Institute, Seoul National University College of Medicine, Seoul, ${ }^{2}$ Nano System Institute, Seoul National University, Seoul, ${ }^{3}$ Department of Obstetrics and Gynecology, Seoul National University College of Medicine, Seoul, Korea, \\ ${ }^{4}$ Departments of Obstetrics \& Gynecology and Cellular \& Molecular Medicine, Interdisciplinary School of Health Sciences, University of Ottawa, and Chronic Disease Program, Ottawa Hospital Research Institute, Ottawa, ON, Canada, ${ }^{5}$ Peggy and Stephenson Cancer Center, University of Oklahoma Health Sciences Center, Oklahoma City, OK, USA, ${ }^{6}$ WCU Biomodulation, Department of Agricultural Biotechnology, Seoul National University, Seoul, Korea}

Correspondence: Yong Sang Song, MD, PhD Department of Obstetrics and Gynecology, Seoul National University College of Medicine, 101 Daehak-ro, Jongno-gu, Seoul 03080, Korea Tel: 82-2-2072-2822

Fax: 82-2-762-3599

E-mail: yssong@snu.ac.kr

Received April 25, 2016

Accepted June 24, 2016

Published Online July 18, 2016

\begin{abstract}
Purpose
Adipose stromal cells (ASCs) play an important regulatory role in cancer progression and metastasis by regulating systemic inflammation and tissue metabolism. This study examined whether visceral and subcutaneous ASCs (V- and S-ASCs) facilitate the growth and migration of ovarian cancer cells.
\end{abstract}

\section{Materials and Methods}

$\mathrm{CD}^{-}{ }^{-}$and $\mathrm{CD} 31^{-}$double-negative ASCs were isolated from the subcutaneous and visceral fat using magnetic-activated cell sorting. Ovarian cancer cells were cultured in conditioned media (CM) obtained from ASCs to determine the cancer-promoting effects of ASCs. A 3-(4,5-dimethylthiazol-2-yl)-2,5-diphenyl tetrazolium bromide (MTT) assay, Boyden chamber assay, and western blotting were performed to determine the proliferative activity, migration ability, and activation of the JAK2/STAT3 pathway, respectively.

\section{Results}

CM from ASCs enhanced the migration of the ovarian cancer line, SKOV3, via activation of the JAK2/STAT3 signaling pathway. Interestingly, in response to ASC-CM, the ascites cells derived from an ovarian cancer patient showed an increase in growth and migration. The migration of ovarian cancer cells was suppressed by blocking the activation of JAK2 and STAT3 using a neutralizing antibody against interleukin 6, small molecular inhibitors (e.g., WP1066 and TG101348), and silencing of STAT3 using siRNA. Anatomical differences between S- and V-ASCs did not affect the growth and migration of the ovarian cancer cell line and ascites cells from the ovarian cancer patients.

\section{Conclusion}

ASCs may regulate the progression of ovarian cancer, and possibly provide a potential target for anticancer therapy.

\section{Introduction}

Ovarian cancer is commonly resistant to chemotherapeutic treatments and is prone to subsequent relapse [1]. Although ovarian cancer is relatively less common among gynecologic cancers, it has one of the highest mortality rates because
Key words

Ovarian neoplasms, Adipose tissue, Interleukin-6,

Cell movement, Adipose stromal cells patients are usually diagnosed at an advanced stage [2] Ovarian cancer disseminates mainly into the peritoneal cavity and abdominal organs including the omentum via the physiological movement of peritoneal fluid [3,4]. Given the epidemiological data indicating that $80 \%$ of patients with serious ovarian carcinoma show abdominal dissemination including the omentum, the intra-abdominal fat is believed 
to act as a major regulator of ovarian cancer metastasis [4-6]. Fat tissue has been reported to be a source of cytokines, which stimulates tumor growth and migration, and metabolites to meet the high-energy demands of tumors [6,7]. Therefore, understanding the molecular mechanisms through which fat depots regulate the phenotype of ovarian cancer cells may provide important clues for the development of anticancer therapies for this disease.

Fat tissue consists of a range of cell types, including mature adipocytes, pre-adipocytes, immune cells, endothelial cells, and adipose stromal cells (ASCs) [8]. ASCs resemble bone marrow mesenchymal stem cells (MSCs), as indicated by their surface markers and differentiation potential into mesenchymal lineages. Accumulating evidence suggests that ASCs as well as adipocytes, endothelial cells, and macrophages in fat tissue can be a major source of cytokines and chemokines [9-11]. ASCs produce and secrete specific growth factors and pro-inflammatory cytokines, such as interleukin6 , hematopoietic colony-stimulating factors, and macrophage colony-stimulating factor, which promote cell proliferation, vascularization, and angiogenesis in endometrial, breast, and prostate cancers [12-14]. Therefore, ASCs may be linked to the progression of cancer by producing a more attractive microenvironment for tumor growth. On the other hand, the relationship between ASCs and ovarian cancer and the molecular mechanisms underlying any interaction between these cells have not been established. This paper reports that ASCs are involved in promoting the growth and migration of ovarian cancer cells via activation of the interleukin 6 (IL-6)/JAK2 / STAT3 pathway.

\section{Materials and Methods}

\section{Isolation of $\mathrm{CD} 45^{-} / \mathrm{CD} 31^{-} \mathrm{ASCs}$}

All protocols were reviewed and approved by the Seoul National University Hospital Institutional Review Board. Human subcutaneous and visceral adipose tissues were surgically obtained from four patients with benign urologic or gynecologic diseases. The adipose tissues were dissociated $\left(37^{\circ} \mathrm{C}, 1\right.$ hour) with collagenase type IA solution (SigmaAldrich, St. Louis, MO). The cells were centrifuged (500 $\times \mathrm{g}$, 4 minutes) and the stromal vascular fraction (SVF) was collected as a pellet. The SVF was treated with ammonium-chloride-potassium lysing buffer (Lonza, Muenchsteiner-strasse, Switzerland) at room temperature for 2 minutes, and then centrifuged. The resulting cell pellet was diluted with a magnetic-activated cell sorting (MACS) buffer (MiltenyiBiotec, Teterow, Germany). To avoid contamination with hemato- poietic stem cells and endothelial cells, the cells were incubated (room temperature, 15 minutes) with anti-CD45 and anti-CD31 antibodies attached to the microbeads (BD Biosciences, San Jose, CA). The CD45- and CD31-negative cells were collected from the SVF using MACS (Fig. 1A).

\section{Culture of ASCs and ovarian cancer cells}

The human ovarian cancer cell line, SKOV3, was purchased from the American Type Culture Collection (Rockville, MD). The ASCs were cultured in Dulbecco's modified Eagle medium (DMEM)/F12 (Life Technologies, Gaithersburg, MD) supplemented with $10 \%$ fetal bovine serum (FBS; Life Technologies), $100 \mathrm{U} / \mathrm{mL}$ penicillin, and $100 \mathrm{~g} / \mathrm{mL}$ streptomycin (1\% PS; Life Technologies) [9]. The SKOV3 cells were cultured in the same culture medium as that of the ASCs to minimize the differences caused by the different culture media. The cells were maintained at $37^{\circ} \mathrm{C}$ in a humidified atmosphere of $5 \% \mathrm{CO}_{2}$.

\section{Isolation and culture of ascites cells from an ovarian can- cer patient}

Ascites aspirated from an ovarian cancer patient at an advanced stage was diluted with an equal volume of phosphate buffered saline (PBS) and centrifuged $\left(4^{\circ} \mathrm{C}, 1,400 \times \mathrm{g}\right.$, 30 minutes). The cells were gently overlaid onto FicollPaque-PREMIUM and centrifuged $\left(4^{\circ} \mathrm{C}, 1,400 \times \mathrm{g}, 30 \mathrm{~min}-\right.$ utes). The cell layer was washed with PBS and suspended in DMEM/F12 supplemented with 10\% FBS and 1\% PS. The ascites cells were maintained at $37^{\circ} \mathrm{C}$ in a humidified atmosphere of $5 \% \mathrm{CO}_{2}$.

\section{Immunophenotype of ASCs}

The ASCs $\left(1 \times 10^{5}\right.$ cells $)$ were incubated with the antibodies or matching isotype control (20 minutes, $\left.4^{\circ} \mathrm{C}\right)$. After washing the cells, propidium iodide was added to identify the dead cells. The labeled cells were isolated using a FACSCanto II flow cytometer, and analyzed using BD FACS Diva software (BD Biosciences). The antibodies were phycoerythrin (PE)conjugated mouse anti-CD45, -CD90, -CD73, and -CD166, and fluorescein isothiocyanate (FITC)-conjugated mouse anti-HLA-DA, -CD31, -CD34, and -CD105. The isotype con-

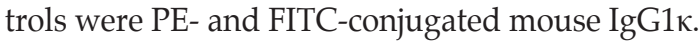

\section{In vitro differentiation of ASCs into adipogenic, chon- drogenic, and osteogenic lineage}

The ASCs were seeded onto 12 -well plates $\left(1 \times 10^{5}\right.$ cells / well) and cultured in an induction medium. For adipogenic differentiation, the subcutaneous and visceral ASCs (S- and 


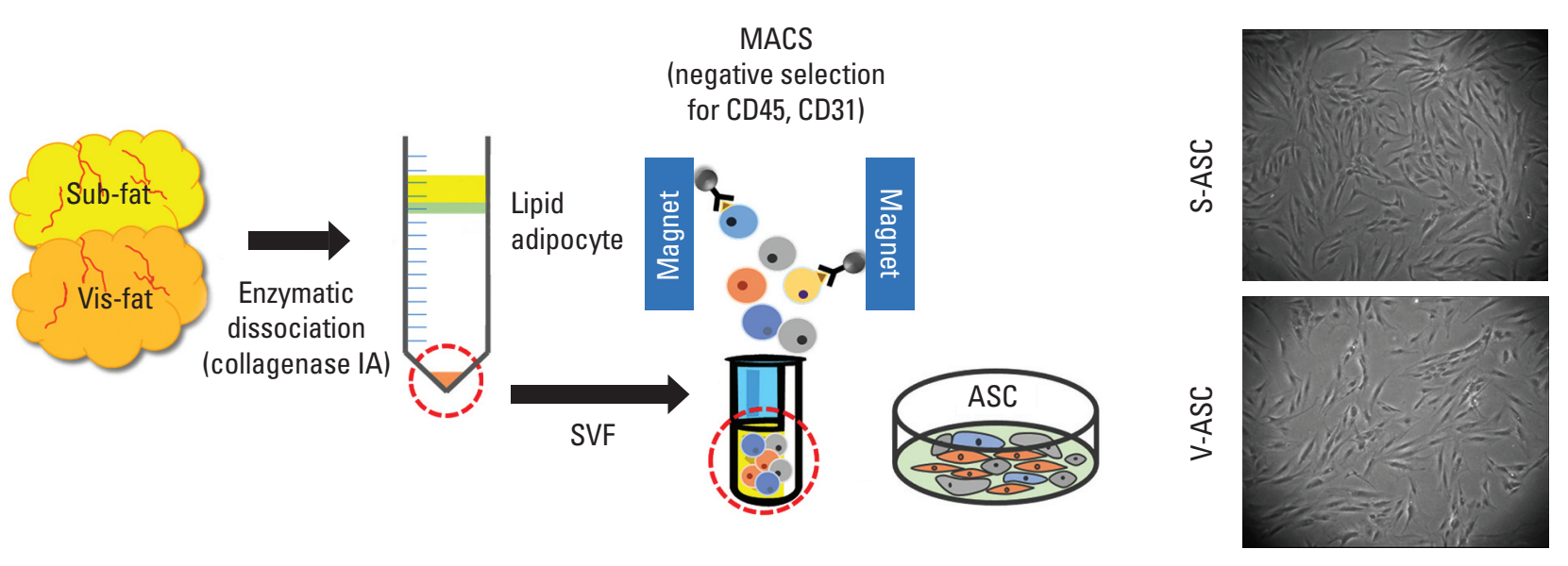

C
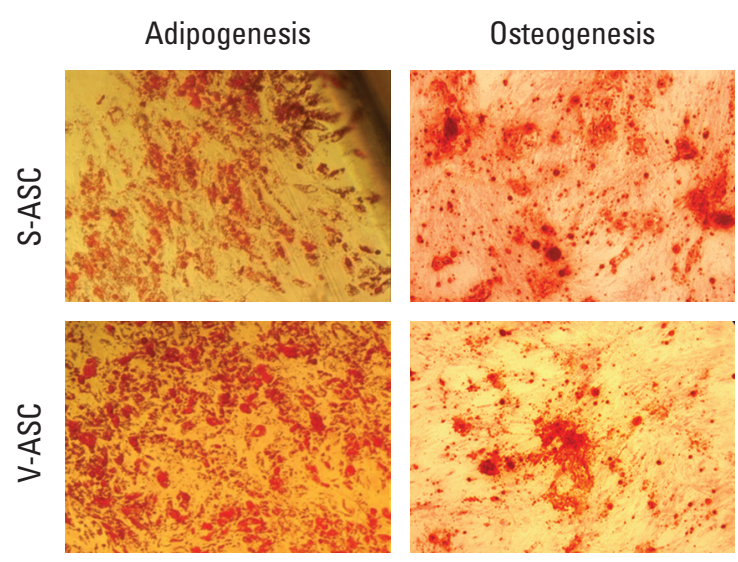

Chondrogenesis
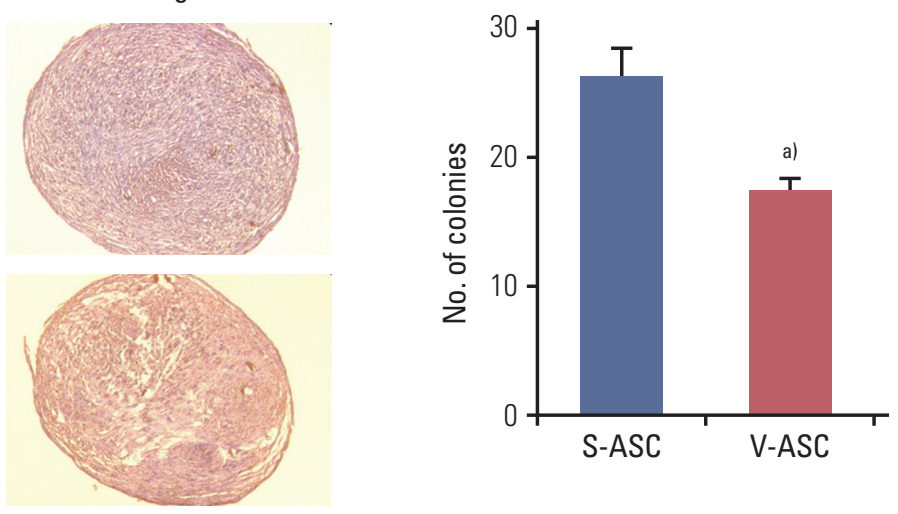

Fig. 1. ASCs isolated from human subcutaneous and visceral fat. (A) The SVF was isolated from subcutaneous and visceral fat tissue. To remove the endothelial and hematopoietic cells from the SVF, CD31- and CD45-negative ASCs were isolated by MACS. (B) The morphologies of S- and V-ASCs were evaluated by an inverted phase-contrast microscope ( $\times 40$ objective) at passage 2. (C) The S- and V-ASCs were able to differentiate into adipogenic, osteogenic, and chondrogenic lineages under induction conditions. (D) One hundred S- and V-ASCs were cultured on a 100-mm plate for 7 days, and the number of clones was counted after staining with $0.5 \%$ crystal violet. ASC, adipose stromal cell; SVF, stromal vascular fraction; MACS, magnetic-activated cell sorting; S-ASC, subcutaneous adipose stromal cell; V-ASC, visceral adipose stromal cell. a) $\mathrm{p}<0.05$, S-ASC vs. V-ASC.

V-ASCs) were incubated in an adipogenic induction medium consisting of DMEM/F12, $0.5 \mathrm{mM}$ isobutylmethylxanthine (Sigma-Aldrich), $50 \mu \mathrm{M}$ indomethacin (Sigma-Aldrich), $5 \mathrm{mM}$ dexamethasone (Sigma-Aldrich), 10\% FBS, and 1\% PS. The medium was changed every second day and the cells were cultured for 3 weeks. Oil Red O staining was performed to visualize the lipid droplets. For chondrogenic differentiation, S- and V-ASCs $\left(1 \times 10^{6}\right.$ cells) were cultured in a chondro- genic induction medium, consisting of DMEM (HyClone, Logan, UT) containing $500 \mathrm{ng} / \mathrm{mL}$ bone morphogenetic protein 6 (R\&D Systems, Minneapolis, MN), $10 \mathrm{ng} / \mathrm{mL}$ transforming growth factor $\beta 3$ (R\&D Systems), $100 \mathrm{nM}$ dexamethasone, $50 \mu \mathrm{g} / \mathrm{mL}$ ascorbate-2-phosphate (Sigma-Aldrich), $40 \mu \mathrm{g} / \mathrm{mL}$ proline (Sigma-Aldrich), $100 \mu \mathrm{g} / \mathrm{mL}$ pyruvate (Sigma-Aldrich), $1 \times$ mixture of recombinant human insulin, transferrin, and sodium selenite liquid media 
(Sigma-Aldrich), 10\% FBS, and 1\% PS. After 3 weeks, deposition of sulfated glycosaminoglycans was detected with toluidine blue. For osteogenic differentiation, the cells were cultured in an osteogenic induction medium consisting of DMEM/F12 supplemented with $1 \mathrm{nM}$ dexamethasone, 10 $\mathrm{mM} \beta$-glycerolphosphate (Sigma-Aldrich), $50 \mu \mathrm{M}$ ascorbate2-phosphate, 10\% FBS, and 1\% PS. After 3 weeks, calcium accumulation was visualized with Alizarin Red S.

\section{Measurement of relative cell growth}

The conditioned medium $(\mathrm{CM})$ was collected from $2 \times 10^{3}$ cells $/ \mathrm{cm}^{2}$ of ASCs cultured for 48 hours. The SKOV3 cells were seeded onto 96-well plates to determine their cell growth in S-ASC-CM, V-ASC-CM, and control medium. The proliferation of SKOV3 and ascites cells in S-ASC-CM and V-ASC-CM was compared with the control medium. After plating for 24 hours, the ascites cells were cultured in S-ASC-CM and V-ASC-CM and control medium for 7 days. The cultured cells were treated $\left(37^{\circ} \mathrm{C}, 3\right.$ hours $)$ with $3-(4,5-$ dimethylthiazol-2-yl)-2,5-diphenyl tetrazolium bromide (MTT, $50 \mu \mathrm{L}$; Amresco, Solon, OH). After incubation, MTT was solubilized with dimethyl sulfoxide at room temperature for 30 minutes. The absorbance was measured spectrophotometrically at $540 \mathrm{~nm}$.

\section{Wound healing assay}

The SKOV3 cells were cultured in 6-well plates for 24 hours. After the complete medium was changed to ASCs-CM or control medium, the cell layer was scraped with a pipette tip to produce a wound. The wound closure distance was measured using ImageJ software.

\section{Migration assay}

To assess the influence of the ASCs on the migratory ability of SKOV3 and ascites cells, the Boyden chamber with a transparent PET membrane ( $8 \mu \mathrm{m}$ pore size; BD Biosciences) was used. The cancer cells were seeded onto the inserts, and the inserts were then transferred to ASC-CM or control medium. After 24 hours ( 48 hours for ascites cells), the migratory cells were fixed with $4 \%$ formaldehyde, and stained with $0.5 \%$ crystal violet.

\section{Depletion of secreted IL-6}

The control medium and ASCs-CM were incubated with $500 \mathrm{ng} / \mathrm{mL}$ of a goat polyclonal anti-recombinant human (rh) IL-6 (R\&D Systems, Minneapolis, MN) or a normal goat IgG control (R\&D Systems) on a shaker at $4^{\circ} \mathrm{C}$ for 3 hours. The media were used in the migration assays, as described above.

\section{Western blotting}

The cultured SKOV3 were harvested and lysed with the protein extraction buffer. The proteins were separated on a $6 \%$ sodium dodecyl sulfate polyacrylamide gel electrophoresis and transferred to a nitrocellulose membrane. The membrane was blocked with 5\% skim milk in Tris-buffered saline containing $0.1 \%$ Tween 20 (TBS-T) and incubated (overnight, $4^{\circ} \mathrm{C}$ ) with the primary antibodies. After washing three times with TBS-T, the membrane was incubated with peroxidaseconjugated secondary antibody and visualized using a western blot detection kit (Ab Frontier, Seoul, Korea).

\section{Statistical analysis}

The data are presented as mean \pm standard error of mean of four ASC samples $(n=4)$. One-way ANOVA and a Student's $\mathrm{t}$ test were used for statistical analyses. Significant differences among the experimental groups were analyzed using Scheffe's post hoc test. All analyses were conducted using IBM SPSS ver. 20 (IBM Co., Armonk, NY).

\section{Results}

\section{ASCs isolated from subcutaneous and visceral fat depots share similar features to MSCs}

S- and V-ASCs exhibited a similar morphology to that of MSCs with a fibroblast-like shape (Fig. 1B). The adipogenic, osteogenic, and chondrogenic differentiation assays were performed to confirm the differentiation abilities of ASCs. S- and V-ASCs formed lipid droplets, sulfated glycosaminoglycans, and accumulated calcium in the cytoplasm under the adipogenic, chondrogenic, and osteogenic differentiation conditions, respectively (Fig. 1C). No differences in morphology and differentiation ability were observed between S- and V-ASCs. On the other hand, the colony-forming ability of S-ASCs was higher than V-ASCs (Fig. 1D). S- and V-ASCs expressed CD73, CD90, CD105, and CD166, but did not express the hematopoietic and endothelial cell markers (CD45 and CD31), CD34, and HLA-DR (Fig. 2). These results show that CD45 and CD31-negative ASCs have similar characteristics to the MSCs, and subcutaneous fat contains more ASCs than visceral fat. 
S-ASC
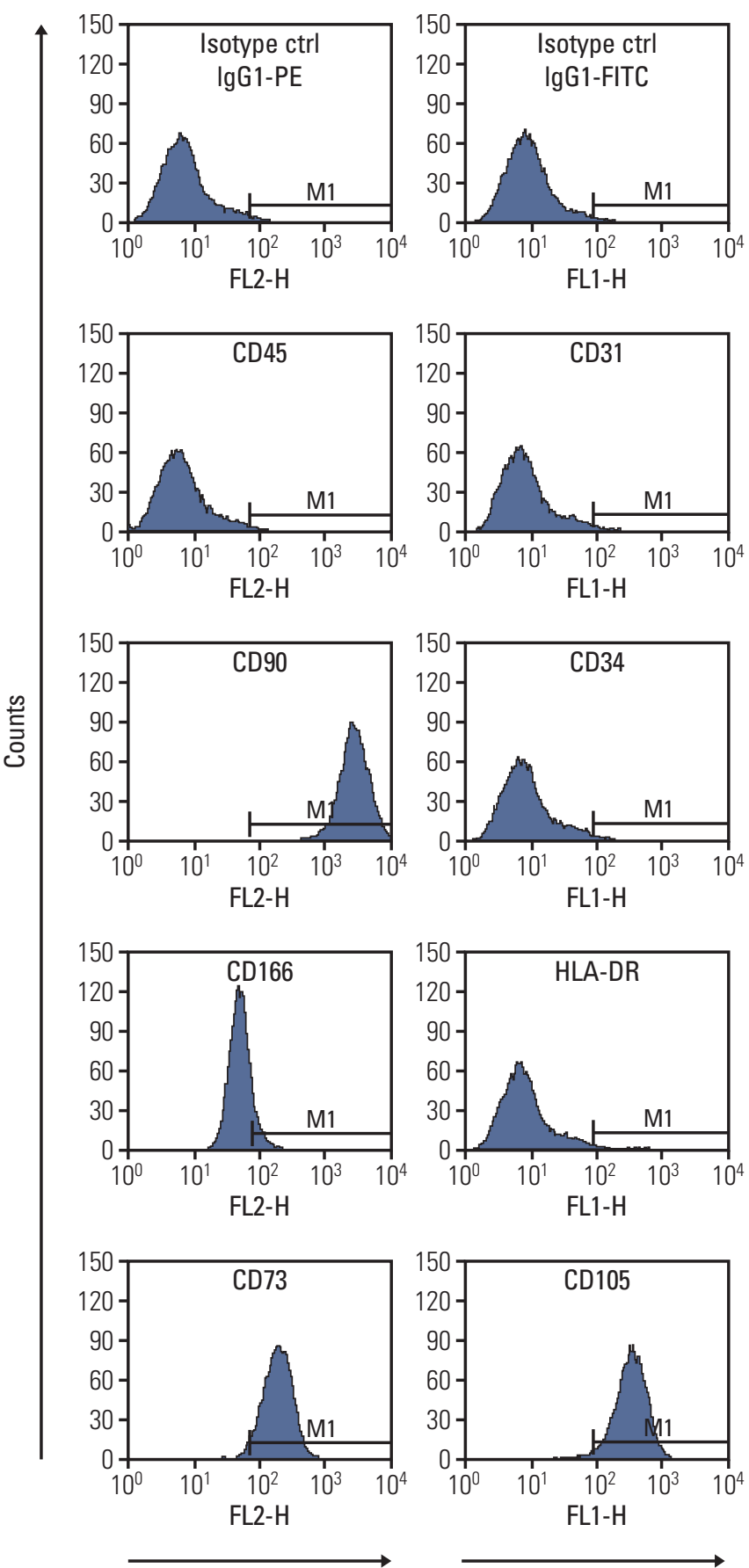

PE

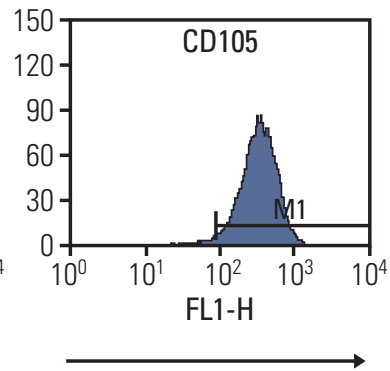

FITC

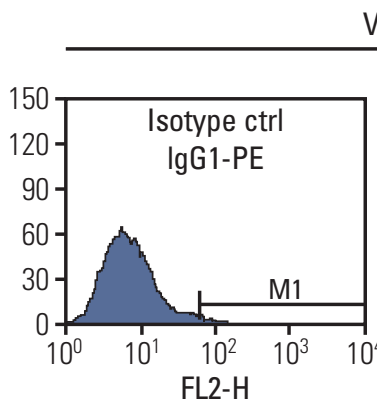

V-ASC
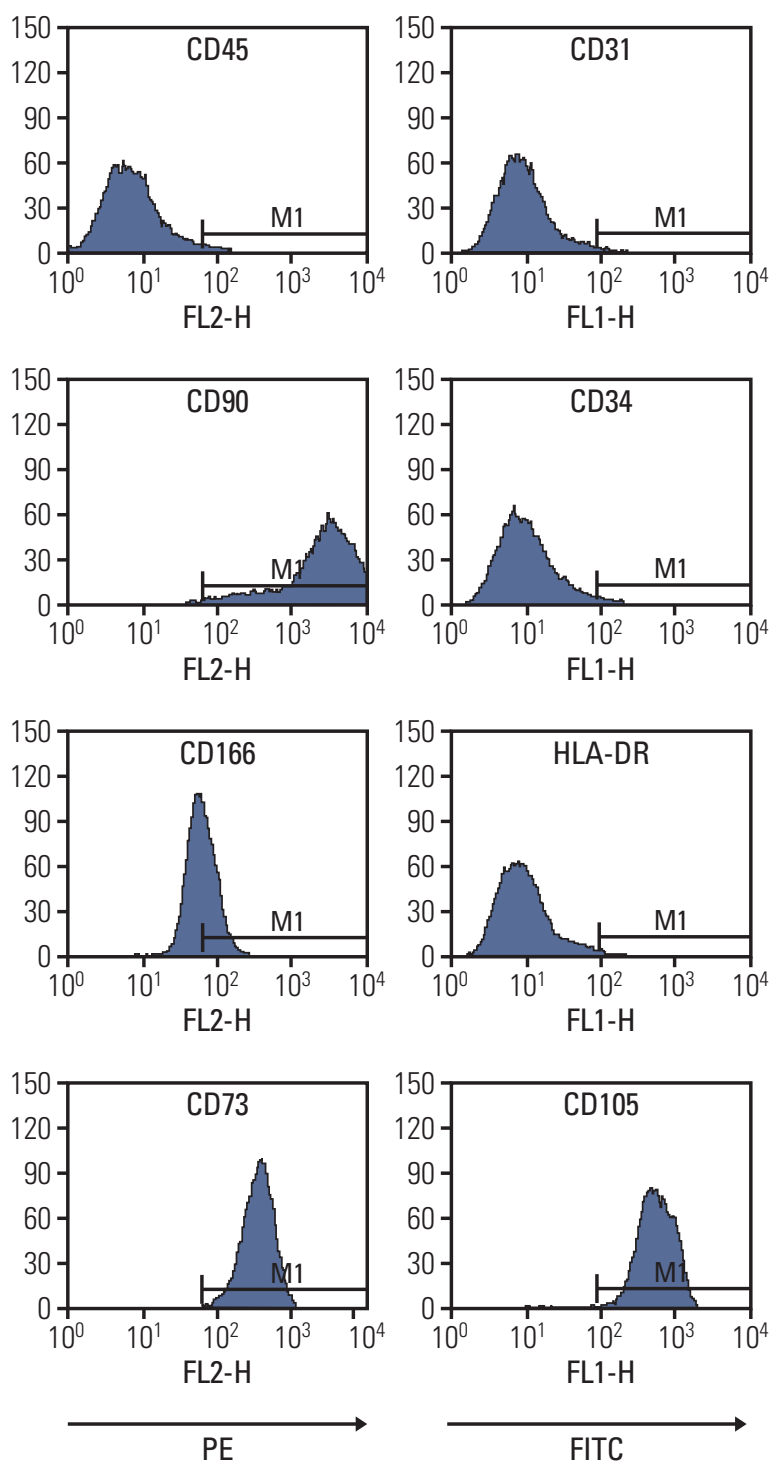

Fig. 2. S- and V-ASCs express specific surface markers of MSCs. The S- and V-ASCs were labeled with CD45, CD73, CD90, and CD166 antibodies conjugated to PE and with CD31, CD34, CD105, and HLA-DR antibodies conjugated to FITC at passage 3. The labeled ASCs were analyzed by flow cytometry. IgG1 $\kappa$-conjugated to PE and to FITC were used as the isotype controls. Both S- and V-ASCs expressed CD90, CD166, CD73, and CD105, but did not express CD45, CD31, CD34, and HLA-DR. S-ASC, subcutaneous adipose stromal cell; V-ASC, visceral adipose stromal cell; MSC, mesenchymal stem cell; PE, phycoerythrin; FITC, fluorescein isothiocyana. 


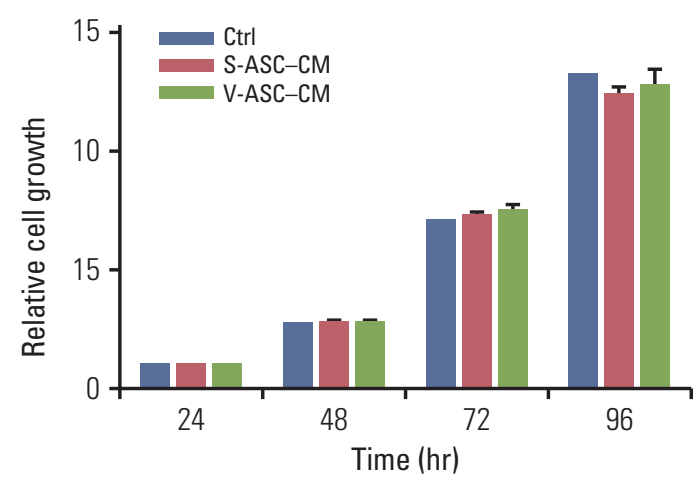

B
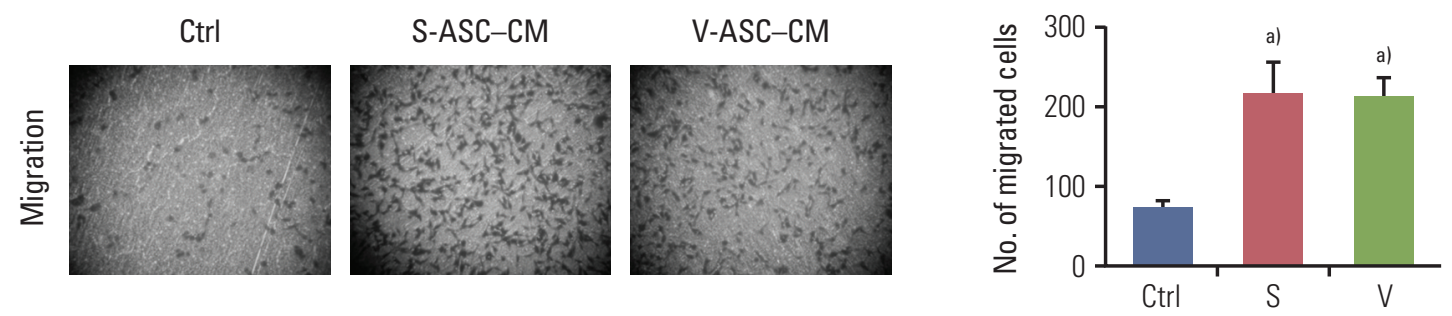

C

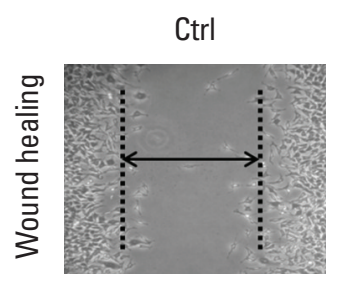

S-ASC-CM

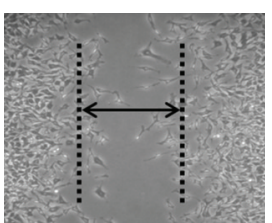

V-ASC-CM

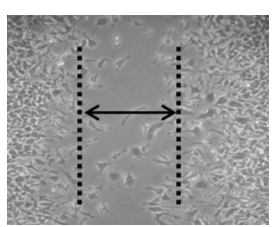

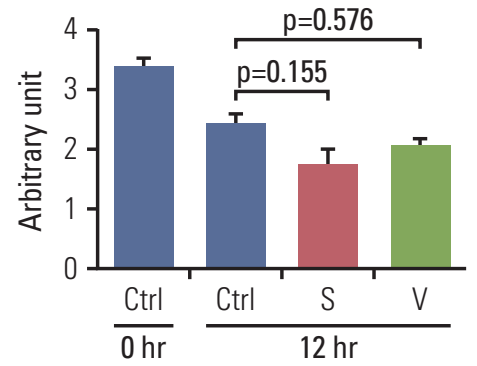

Fig. 3. Conditioned media from S- and V-ASCs promote the migration of ovarian cancer cells. (A) SKOV3 cells were cultured in S-ASC-CM and V-ASC-CM for 24, 48, 72, and 96 hours. The relative cell growth of the SKOV3 cells in S-ASC-CM and V-ASC-CM compared to the control medium was determined using an MTT assay. (B) The migration of SKOV3 cells was determined using a Boyden chamber assay. (C) The wound healing ability of SKOV3 cells was verified by measuring the wound closure distance in 12 hours. S-ASC, subcutaneous adipose stromal cell; V-ASC, visceral adipose stromal cell; CM, conditioned media; MTT, 3-(4,5-dimethylthiazol-2-yl)-2,5-diphenyl tetrazolium bromide; S, S-ASC-CM; V, V-ASC-CM. ${ }^{a} \mathrm{p}<0.05$, control vs. $S$ vs. V.

2. ASC-CM enhances the migratory ability of ovarian cancer cells with no difference between S-ASC-CM and V-ASC-CM

The proliferation of SKOV3 cells was not influenced by the presence of S-ASC-CM and V-ASC-CM compared to control the medium (Fig. 3A). However, the SKOV3 cells migrated more readily across the membrane of the insert in S-ASC$\mathrm{CM}$ and V-ASC-CM than in the control medium (Fig. 3B), and the wound healing ability was also increased in ASCCM relative to the control (Fig. 3C). Overall, these results suggest that certain paracrine factors secreted from S- and V- 

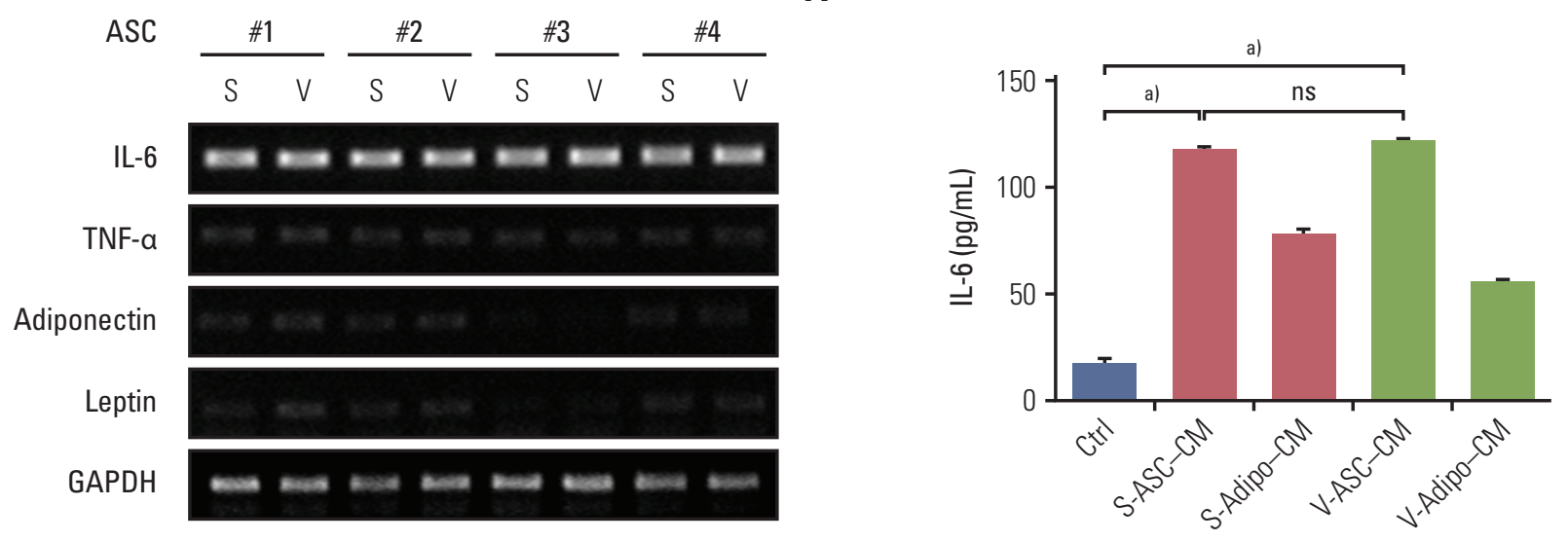

C
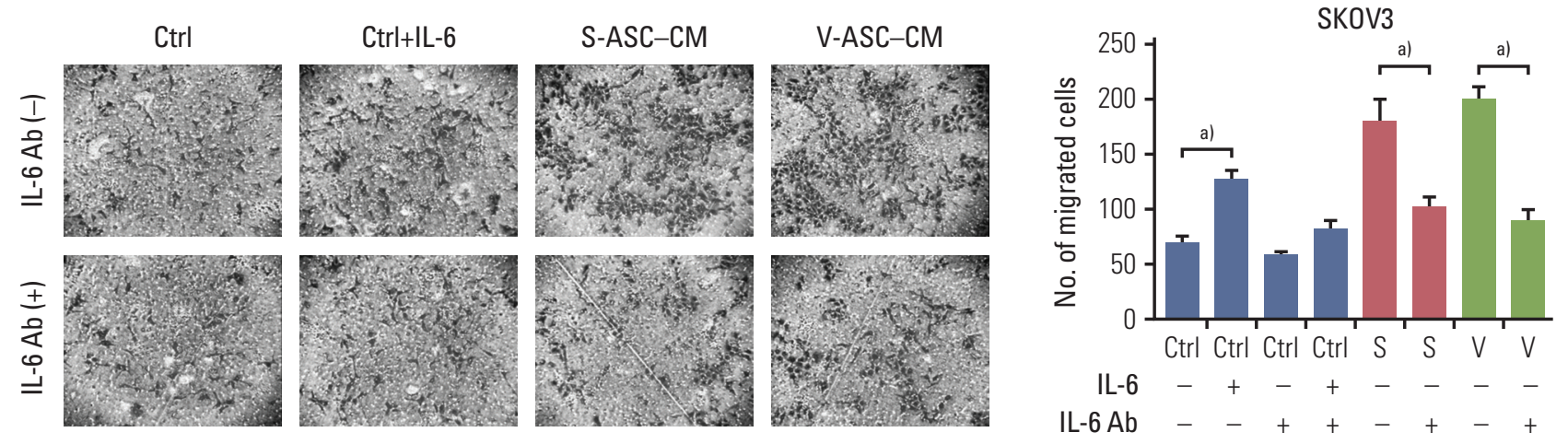

Fig. 4. S- and V-ASCs enhance the migration of ovarian cancer cells through an IL-6-mediated pathway. (A) Messenger RNA expression of adipokines including IL-6, TNF- $\alpha$, adiponectin, and leptin was measured by RT-PCR. GAPDH was used as a reference gene. (B) An ELISA assay was conducted to detect the secretory IL-6 present in the control medium, S-ASC$\mathrm{CM}, \mathrm{V}-\mathrm{ASC}-\mathrm{CM}$, and subcutaneous and visceral differentiated adipocytes (S- and V-adipo-CM). Complete medium was used as a control. (C) SKOV3 cells were cultured in S-ASC-CM and V-ASC-CM with or without anti-IL-6 Ab (500 ng/mL). The migration ability was determined using a Boyden chamber assay. Control medium with rh-IL-6 $(50 \mathrm{ng} / \mathrm{mL})$ was used as a positive control. S-ASC, subcutaneous adipose stromal cell; V-ASC, visceral adipose stromal cell; IL-6, interleukin 6; TNF- $\alpha$, tumor necrosis factor $\alpha$; RT-PCR, reverse transcription polymerase chain reaction; GAPDH, glyceraldehyde 3-phosphate dehydrogenase; ELISA, enzyme-linked immunosorbent assay; CM, conditioned media; rh, recombinant human; $\mathrm{S}$, S-ASC-CM; V, V-ASC-CM; ns, not significant; Ctrl, control. a) $\mathrm{p}<0.05$.

ASCs may regulate the migration of ovarian cancer cells, but the difference in their fat origin does not affect the migration of ovarian cancer cells.

\section{IL-6 enhances the migration of ovarian cancer cells}

The mRNA expression of several adipokines including IL-6, tumor necrosis factor $\alpha$, adiponectin, and leptin were screened to determine the cancer-promoting effects of ASCs. As shown in Fig. 4A, IL-6 was strongly expressed in S- and
V-ASCs, but the expression of the other adipokines was either marginal or undetectable. The concentration of secretory IL-6 was higher in ASC-CM than in adipocytes CM and control medium (Fig. 4B). However, there was no difference between the S- and V-ASCs with respect to their effects on the proliferation and migration of cancer cells. To determine if IL-6 present in ASC-CM influences the migratory ability of SKOV3 cells, the anti-IL-6 antibody was added to ASC-CM. The anti-IL- 6 antibody decreased the number of migratory cells present at the bottom of the Boyden chamber inserts 
A

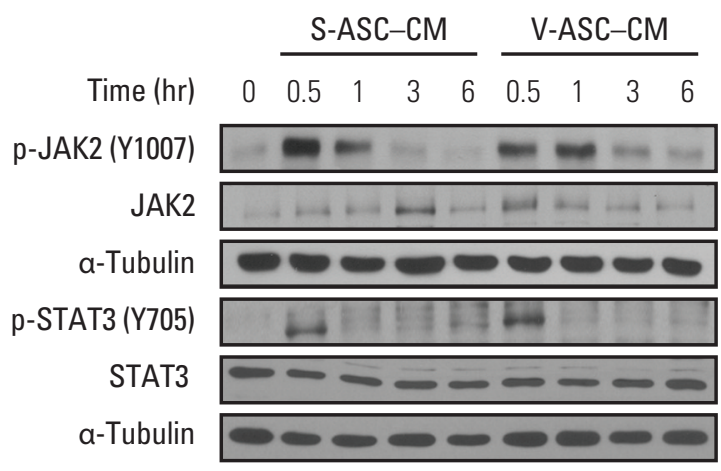

B

C
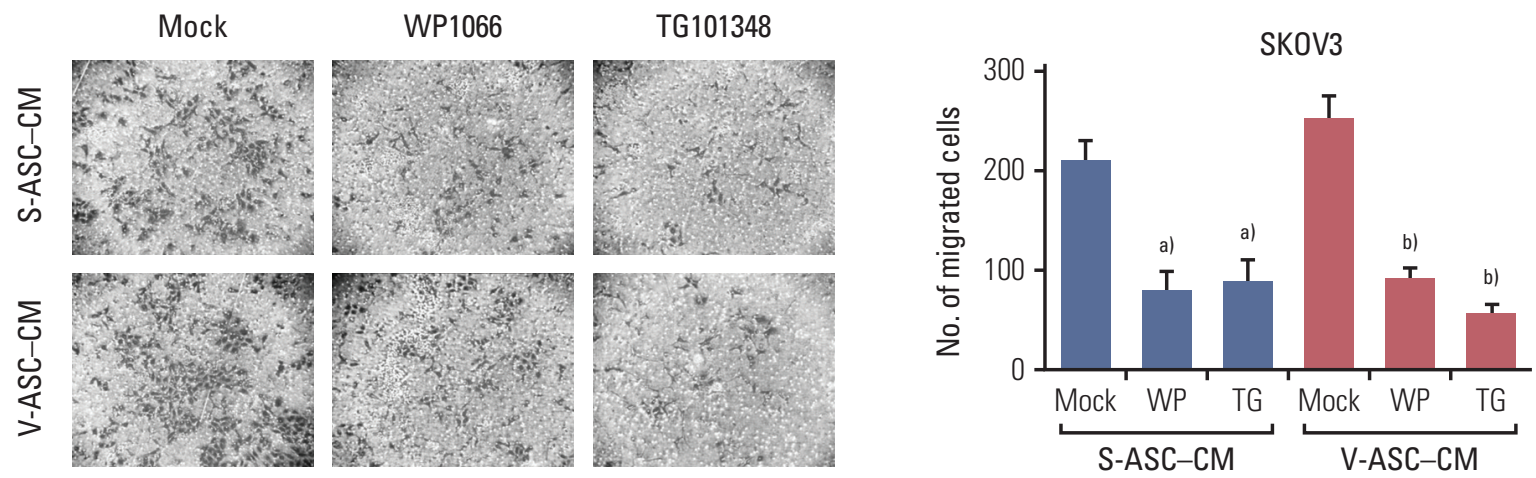

D
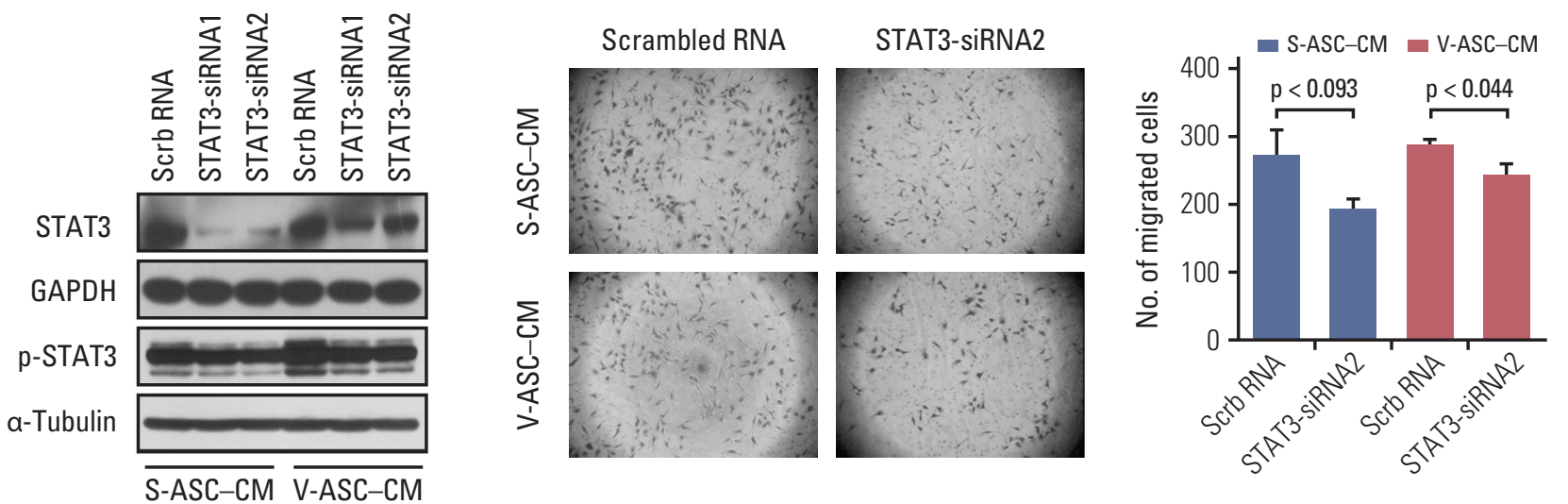

Fig. 5. Activation of JAK2 and STAT3 in response to IL-6 from ASCs increases the migration of ovarian cancer cells. (A) The total JAK2, p-JAK2 (Y1007), total STAT3, and p-STAT3 (Y705) were determined by western blot analysis. (B) After the depletion of IL-6 using anti-IL-6 Ab (500 ng / mL), the total JAK2, p-JAK2, STAT3, and p-STAT3 were analyzed by western blot. The control medium with rh-IL-6 (50 ng/mL) was used as a positive control. (C) After the SKOV3 cells were incubated in S-ASC-CM and V-ASC-CM with the addition of $2 \mu \mathrm{M}$ WP1066 or $1 \mu \mathrm{M}$ TG101348 for 12 hours, a Boyden chamber assay was conducted. (D) Silencing of STAT3 using siRNA reduces the migration of SKOV3. SKOV3 was transfected with Scrb RNA (100 $\mathrm{nM}$ ) and siRNA for STAT3 (STAT3-siRNA1 and 2, $100 \mathrm{nM}$ ), and cultured in S-ASC-CM and V-ASC-CM for the migration assay. The expression of p-STAT3 and total STAT3 was inhibited after the silencing of STAT3 using siRNA. (E) After the inhibition of STAT3 using siRNA2, the migratory cells were determined by a Boyden chamber assay. (Continued to the next page) 


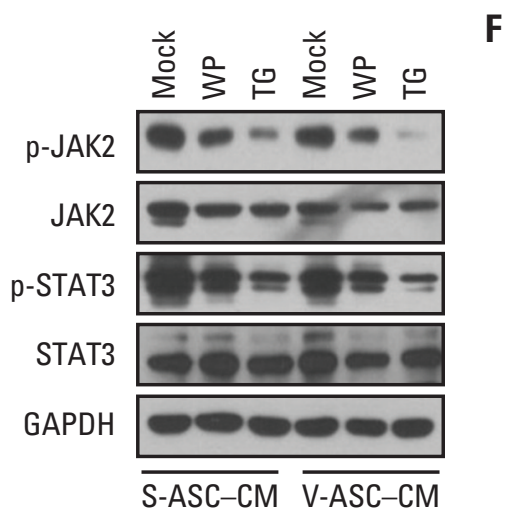

Fig. 5. (Continued from the previous page) (F) After treating SKOV3 with S-ASC-CM and V-ASC-CM containing either $1 \mu \mathrm{M}$ WP1066 or $2 \mu \mathrm{M}$ TG101348, the inhibition of p-JAK2, total JAK2, p-STAT3, and total STAT3 protein levels was confirmed by western blot analysis. IL-6, interleukin 9; ASC, adipose stromal cell; rh, recombinant human; S-ASC, subcutaneous adipose stromal cell; V-ASC, visceral adipose stromal cell; $\mathrm{CM}$, conditioned media; Scrb RNA, scrambled RNA; GAPDH, glyceraldehyde 3-phosphate dehydrogenase. a) $p<0.05$, mock vs. WP vs. TG in S-ASC-CM, ${ }^{\text {b) }} \mathrm{p}<0.05$, in V-ASC-CM.

(Fig. 4C). These results suggest that IL-6 from the ASCs may play an important role in the migration of ovarian cancer cells.

\section{JAK2/STAT3 signaling is activated by IL-6 secreted from ASCs in ovarian cancer cells}

The activation of the JAK2/STAT3 pathway in response to IL-6 secreted from ASCs was evaluated. The levels of phosphorylated JAK2 and STAT3 in SKOV3 cells, but not the total JAK2 and STAT3, were increased in 30 minutes, and then started to decrease in 1 hour (Fig. 5A). To confirm that the IL-6 from ASCs affects JAK2/STAT3 signaling in SKOV3 cells, an anti-IL-6 neutralizing antibody was added to ASC$\mathrm{CM}$. The addition of IL-6 to the control medium activated JAK2 and STAT3 in the SKOV3 cells as did the ASC-CM, and JAK2/STAT3 activation was attenuated by the anti-IL-6 neutralizing antibody (Fig. 5B). To confirm the relationship between JAK2/STAT3 and the migration of ovarian cancer cells, the SKOV3 cells were treated with a JAK2/STAT3 inhibitor (WP1066) and JAK2 inhibitor (TG101348). Both treatments reduced the number of migratory cells along with the inhibition of phosphorylated JAK2 and STAT3 (Fig. 5C and F). In addition, the inhibition of endogenous STAT3 using siRNA1 and 2 specific to STAT3 was confirmed by western blot analysis (Fig. 5D). Silencing STAT3 using siRNA2 reduced the migration of SKOV3 cells (Fig. 5E). These results support the finding that IL-6 secreted from ASCs may promote the migration of ovarian cancer cells through the activation of JAK2/STAT3.

\section{IL-6/JAK2/STAT3 pathway activated by ASCs enhances the proliferation and migration of ovarian cancer patient- derived ascites cells}

The proliferation of ascites cells was significantly increased in ASC-CM compared to that in the control medium (Fig. 6A). Similar to the SKOV3 cells, ASC-CM increased the migration of ascites cells, which was related to the IL-6/ JAK2/STAT3 pathway (Fig. 6B). The relationship between IL- 6 and the migration of ascites cells was determined by small molecular inhibitors of JAK2 and STAT3 (WP1066 and TG101348). The migration of ascites cells was inhibited when either inhibitor was used (Fig. 6C). Therefore, the ASCs enhance the proliferation and migration of ascites cells via the IL-6/JAK2/STAT3 pathway.

\section{Discussion}

Two types of ASCs were isolated from the subcutaneous and visceral fat depots to determine if the anatomical location of the fat depots affects the functional difference of ASCs because it has been reported that the different physiological effects of S- and V-adipocytes are related to their anatomical distribution [15]. ASCs depending on an individual's fat distribution are distinct with respect to their genome-wide mRNA expression profile, proliferative activity, and differentiation potential $[16,17]$. The genome-wide expression profiles of primary preadipocytes from abdominal subcutaneous, mesenteric, and omental fat depots are also distinct, while their morphological features are similar [18]. On the other hand, ASCs obtained from subcutaneous, visceral, and omental adipose tissue from the same subject share the typical markers with MSCs, and their ultrastructural features and the pattern of cytokine secretion are similar $[16,19]$. In line with previous studies, significant differences in the phenotypic character and in the secretion of cytokines, including IL-6, were not observed between S- and V-ASCs. Therefore, the cancer-promoting stimulation of ASCs may be independent of the fat depot. A possible reason for the discrepancy between these findings and previous studies would be that only IL-6 was focused without excluding the involvement of other inflammatory cytokines/chemokines. Another reason may be related to the use of cellular models derived from 
A
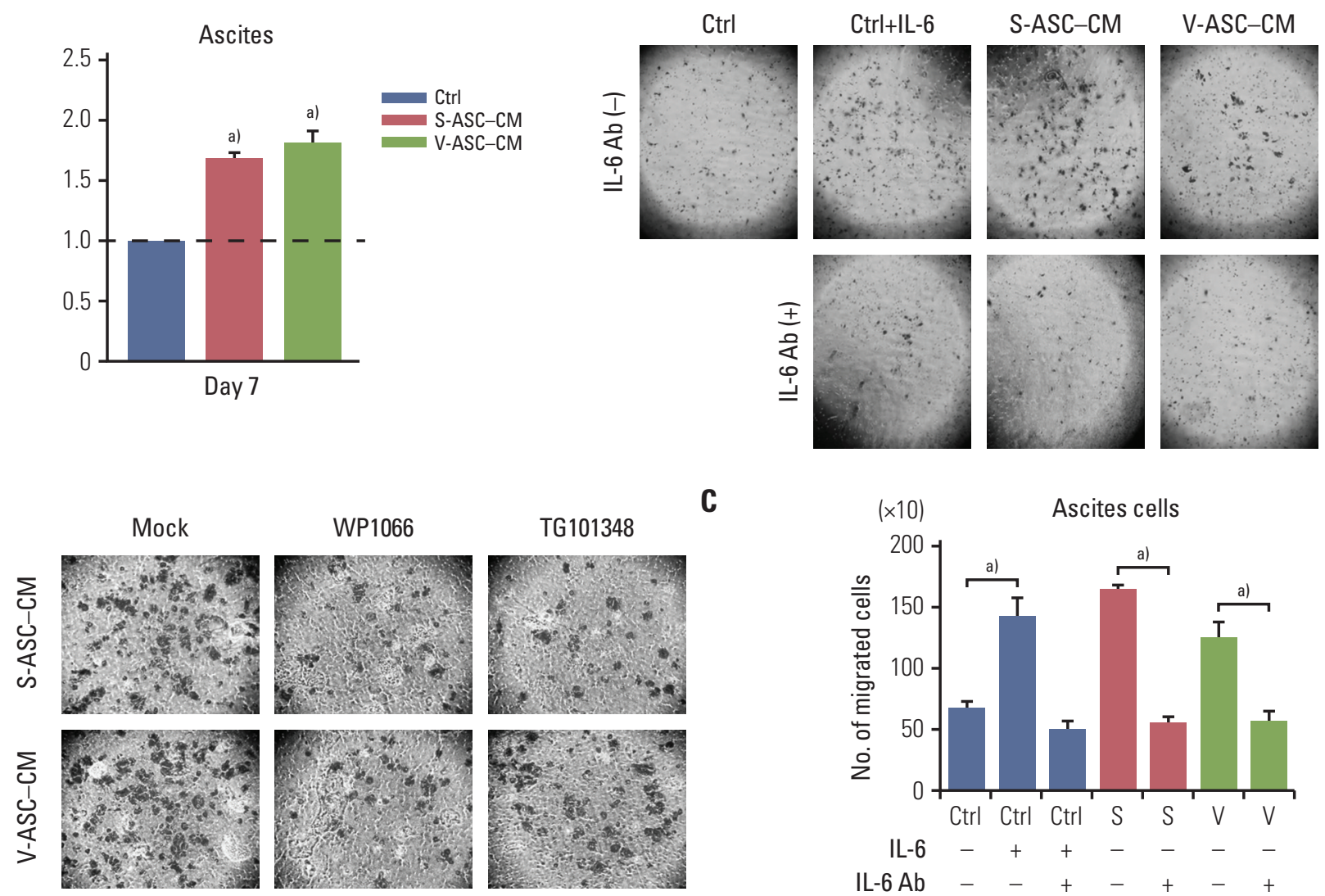

C

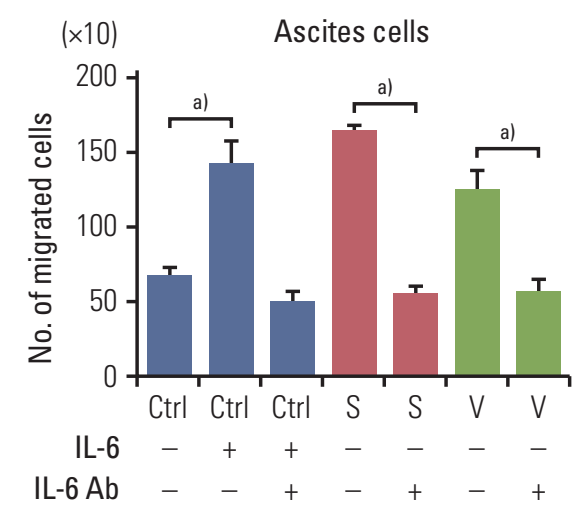

Fig. 6. ASCs enhance the proliferation and migration of ovarian cancer cells isolated from the ascites of an ovarian cancer patient through activation of the IL-6/JAK2/STAT3 pathway. (A) Ascites cells were cultured in S-ASC-CM and V-ASCCM for 7 days. The proliferation of ascites cells was determined by an MTT assay. (B) Ascites cells were cultured in ASC$\mathrm{CM}$ with or without anti-IL-6 Ab (500 ng/mL), and in the control medium with rh-IL-6 (50 ng/mL) and/or anti-IL-6 Ab. After culturing for 24 hours, the number of migratory cells were counted. (C) The ascites cells cultured in ASC-CM for 24 hours were treated with WP1066 and TG101348. Migration was determined using a Boyden chamber assay. ASC, adipose stromal cell; IL-6, interleukin 9; S-ASC, subcutaneous adipose stromal cell; V-ASC, visceral adipose stromal cell; CM, conditioned media; MTT, 3-(4,5-dimethylthiazol-2-yl)-2,5-diphenyl tetrazolium bromide; rh, recombinant human; Ctrl, control; S, S-ASC-CM; V, V-ASC-CM. ${ }^{\text {a) }} \mathrm{p}<0.05$.

subjects with a different body mass index, sex, or other diseases. Visceral adipose tissue with a higher percentage of large adipocytes is functionally distinct from the subcutaneous one in insulin resistance, free fatty acid generation, and glucose uptake [20]. Unlike the differentiated adipocytes, the fate of ASCs is not fully determined because ASCs are undifferentiated cells. Therefore, the physiological functions of S- and V-ASCs may be similar to MSCs rather than adipocytes. These findings showed that S- and V-ASCs share the expression of surface markers, differentiation abilities, and gene expression of adipokines. Moreover, the anatomical origin or distribution of ASCs is not be a critical factor for stimulating the growth or migration of ovarian cancer cells. Therefore, regardless of the ASC origins, a quantitative increase in ASCs by obesity and vice versa could enhance the proliferation or migration of ovarian cancer cells.

ASCs have the features of multipotent MSCs providing tumor cells with a functionally and structurally supportive environment through the establishment of the pre-metastatic niche, causing the induction of the metastatic phenotypes $[21,22]$. ASCs also promote the progression of prostate cancer, and stimulate the migration and invasion of estrogen receptor-negative breast cancer $[12,23]$. Recently, Nowicka et al. [24] suggested that ASCs derived from omental fat tissue 
contribute to the proliferation and migration of ovarian cancer cell lines through the metabolic support of ASCs to ovarian cancer cells. The present findings also showed that $\mathrm{S}$ - and V-ASCs contribute to the increased migration of ascites cells from ovarian cancer patients. Interestingly, ASCs enhanced the proliferation of patient-derived ascites cells, but not SKOV3. SKOV3 is frequently used as an in vitro model instead of primary tumor cells, but Domcke et al. [25] reported that SKOV3 has an overall genomic difference from the high grade serous ovarian cancer. For this reason, there could be a proliferative difference between SKOV3 and ascites cells.

ASCs as well as adipocytes secrete many cytokines and chemokines [9]. The mRNA of IL-6 among several cytokines is strongly expressed in S- and V-ASCs. The IL-6 cytokine family is involved in various biological responses, such as inflammation, immune reaction, and oncogenesis by regulating growth and differentiation. The binding of the ligand to its common receptor subunit gp130 activates the JAK/ STAT pathway, which plays a central role in tumor cell migration. In a previous study, the IL-6 receptor (IL-6R) was strongly expressed in SKOV3 cells and exogenous IL-6 stimulated STAT3 activation, increasing SKOV3 migration. STAT3 was activated through the direct activation of EGFR or IL-6R or the indirect induction of the IL-6R pathway in high-grade ovarian carcinomas [26]. In these findings, the IL-6-induced ovarian cancer cell migration was hampered by blocking IL-6R with an IL-6 antibody or applying specific inhibitors of STAT3 and JAK2, suggesting that IL-6 can play a key role in the proliferation and migration of ovarian cancer cells. ASCs were found to express IL-6 mRNA and secrete the IL-6 protein without any extrinsic stimulation. Based on these results, it was concluded that the enhanced migration and proliferation of ovarian cancer cells is due partially to activation of the JAK2/STAT3 pathway by ASCs-derived IL-6. JAK2 is involved in the progression and drug-response of ovarian cancer. In our previous study, the next-generation sequencing data showed that the JAK/STAT pathways were amplified in primary and metastatic tumor clusters [27]. Other studies also reported that JAK2/STAT3 is activated in high-grade ovarian carcinomas compared to normal ovaries and benign tumors, and is involved in cancer progression and EMT [26]. Moreover, the efficacy of cisplatin is dependent on the JAK2 activity [28]. In addition, it was reported that CA125 is a useful predicting marker of ovarian cancer at advanced stages [29]. This can bind JAK2 and activate STAT3, highlighting the importance of JAK2 in the pathogenesis of CA125-expressing cancers [30]. Therefore, targeting JAK2 using several inhibitors might be an important therapeutic strategy to mitigate the dissemination of ovarian cancer.

\section{Conclusion}

This paper provided direct experimental evidence that IL-6 secreted from ASCs activates the JAK2/STAT3 pathway and contributes to ovarian cancer progression. Targeting both ovarian cancer cells and supportive stromal cells in adipose tissue could be a new approach for ovarian cancer therapy.

\section{Conflicts of Interest}

Conflict of interest relevant to this article was not reported.

\section{Acknowledgments}

This research was supported by the Priority Research Centers Program (2009-0093820), the Pioneer Research Center Program (2012-0009555), the Canadian Institutes of Health Research (MOP-126144), and the National Institutes of Health (CA116984 and CA123233).

\section{References}

1. Jemal A, Bray F, Center MM, Ferlay J, Ward E, Forman D. Global cancer statistics. CA Cancer J Clin. 2011;61:69-90.

2. Lowe KA, Chia VM, Taylor A, O'Malley C, Kelsh M, Mohamed $\mathrm{M}$, et al. An international assessment of ovarian cancer incidence and mortality. Gynecol Oncol. 2013;130:
107-14.

3. Lengyel E. Ovarian cancer development and metastasis. Am J Pathol. 2010;177:1053-64.

4. Naora H. Heterotypic cellular interactions in the ovarian tumor microenvironment: biological significance and thera- 
peutic implications. Front Oncol. 2014;4:18.

5. Naora H, Montell DJ. Ovarian cancer metastasis: integrating insights from disparate model organisms. Nat Rev Cancer. 2005;5:355-66.

6. Nieman KM, Kenny HA, Penicka CV, Ladanyi A, Buell-Gutbrod R, Zillhardt MR, et al. Adipocytes promote ovarian cancer metastasis and provide energy for rapid tumor growth. Nat Med. 2011;17:1498-503.

7. Gilbert CA, Slingerland JM. Cytokines, obesity, and cancer: new insights on mechanisms linking obesity to cancer risk and progression. Annu Rev Med. 2013;64:45-57.

8. Trayhurn P, Beattie JH. Physiological role of adipose tissue: white adipose tissue as an endocrine and secretory organ. Proc Nutr Soc. 2001;60:329-39.

9. Kilroy GE, Foster SJ, Wu X, Ruiz J, Sherwood S, Heifetz A, et al. Cytokine profile of human adipose-derived stem cells: expression of angiogenic, hematopoietic, and pro-inflammatory factors. J Cell Physiol. 2007;212:702-9.

10. Trayhurn P. Endocrine and signalling role of adipose tissue: new perspectives on fat. Acta Physiol Scand. 2005;184:285-93.

11. Wang M, Crisostomo PR, Herring C, Meldrum KK, Meldrum DR. Human progenitor cells from bone marrow or adipose tissue produce VEGF, HGF, and IGF-I in response to TNF by a p38 MAPK-dependent mechanism. Am J Physiol Regul Integr Comp Physiol. 2006;291:R880-4.

12. Prantl L, Muehlberg F, Navone NM, Song YH, Vykoukal J, Logothetis CJ, et al. Adipose tissue-derived stem cells promote prostate tumor growth. Prostate. 2010;70:1709-15.

13. Karnoub AE, Dash AB, Vo AP, Sullivan A, Brooks MW, Bell $\mathrm{GW}$, et al. Mesenchymal stem cells within tumour stroma promote breast cancer metastasis. Nature. 2007;449:557-63.

14. Klopp AH, Zhang Y, Solley T, Amaya-Manzanares F, Marini F, Andreeff M, et al. Omental adipose tissue-derived stromal cells promote vascularization and growth of endometrial tumors. Clin Cancer Res. 2012;18:771-82.

15. Bjorntorp P. The regulation of adipose tissue distribution in humans. Int J Obes Relat Metab Disord. 1996;20:291-302.

16. Baglioni S, Cantini G, Poli G, Francalanci M, Squecco R, Di Franco A, et al. Functional differences in visceral and subcutaneous fat pads originate from differences in the adipose stem cell. PLoS One. 2012;7:e36569.

17. Perrini S, Ficarella R, Picardi E, Cignarelli A, Barbaro M, Nigro $\mathrm{P}$, et al. Differences in gene expression and cytokine release profiles highlight the heterogeneity of distinct subsets of adipose tissue-derived stem cells in the subcutaneous and visceral adipose tissue in humans. PLoS One. 2013;8:e57892.

18. Tchkonia T, Lenburg M, Thomou T, Giorgadze N, Frampton G, Pirtskhalava T, et al. Identification of depot-specific human fat cell progenitors through distinct expression profiles and developmental gene patterns. Am J Physiol Endocrinol Metab. 2007;292:E298-307.

19. Kipps E, Tan DS, Kaye SB. Meeting the challenge of ascites in ovarian cancer: new avenues for therapy and research. Nat Rev Cancer. 2013;13:273-82.

20. Ibrahim MM. Subcutaneous and visceral adipose tissue: structural and functional differences. Obes Rev. 2010;11:11-8.

21. Coffelt SB, Marini FC, Watson K, Zwezdaryk KJ, Dembinski JL, LaMarca HL, et al. The pro-inflammatory peptide LL-37 promotes ovarian tumor progression through recruitment of multipotent mesenchymal stromal cells. Proc Natl Acad Sci U S A. 2009;106:3806-11.

22. Lis R, Touboul C, Mirshahi P, Ali F, Mathew S, Nolan DJ, et al. Tumor associated mesenchymal stem cells protects ovarian cancer cells from hyperthermia through CXCL12. Int J Cancer. 2011;128:715-25.

23. Walter M, Liang S, Ghosh S, Hornsby PJ, Li R. Interleukin 6 secreted from adipose stromal cells promotes migration and invasion of breast cancer cells. Oncogene. 2009;28:2745-55.

24. Nowicka A, Marini FC, Solley TN, Elizondo PB, Zhang Y, Sharp HJ, et al. Human omental-derived adipose stem cells increase ovarian cancer proliferation, migration, and chemoresistance. PLoS One. 2013;8:e81859.

25. Domcke S, Sinha R, Levine DA, Sander C, Schultz N. Evaluating cell lines as tumour models by comparison of genomic profiles. Nat Commun. 2013;4:2126.

26. Colomiere M, Ward AC, Riley C, Trenerry MK, CameronSmith D, Findlay J, et al. Cross talk of signals between EGFR and IL-6R through JAK2/STAT3 mediate epithelial-mesenchymal transition in ovarian carcinomas. Br J Cancer. 2009; 100:134-44.

27. Lee JY, Yoon JK, Kim B, Kim S, Kim MA, Lim H, et al. Tumor evolution and intratumor heterogeneity of an epithelial ovarian cancer investigated using next-generation sequencing. BMC Cancer. 2015;15:85.

28. Song H, Sondak VK, Barber DL, Reid TJ, Lin J. Modulation of Janus kinase 2 by cisplatin in cancer cells. Int J Oncol. 2004; 24:1017-26.

29. Kim HS, Choi HY, Lee M, Suh DH, Kim K, No JH, et al. Systemic inflammatory response markers and CA-125 levels in ovarian clear cell carcinoma: a two center cohort study. Cancer Res Treat. 2016;48:250-8.

30. Lakshmanan I, Ponnusamy MP, Das S, Chakraborty S, Haridas D, Mukhopadhyay P, et al. MUC16 induced rapid G2/M transition via interactions with JAK2 for increased proliferation and anti-apoptosis in breast cancer cells. Oncogene. 2012; 31:805-17. 